\title{
Shape and force analysis of capillary bridge between two slender structured surfaces
}

\author{
Z. F. Zhu ${ }^{1}$, J. Y. Jia ${ }^{1}$, H. Z. Fu ${ }^{2}$, Y. L. Chen ${ }^{1}$, Z. Zeng ${ }^{1}$, and D. L. Yu ${ }^{1}$ \\ ${ }^{1}$ School of Mechano-Electronic Engineering, Xidian University, Xi' an, China \\ ${ }^{2}$ ZTE Corporation, Shenzhen, China \\ Correspondence to: Z. F. Zhu (zhaofeizhu@ stu.xidian.edu.cn)
}

Received: 18 April 2015 - Revised: 1 August 2015 - Accepted: 18 September 2015 - Published: 1 October 2015

\begin{abstract}
When a capillary bridge of a constant volume is formed between two surfaces, the shape of the liquid bridge will change as the separation between those surfaces is varied. To investigate the variable forces and Laplace pressure of the capillary bridge, as the shape the bridge evolves, a pseudo-three-dimensional force model of the capillary bridge is developed. Based on the characteristics of the slender structured surface, an efficient method is employed to directly solve the differential equations defining the shape of the capillary bridge. The spacing between the plates satisfying the liquid confined within the hydrophobic region of the structured surface is calculated. The method described in this paper can prevent meshing liquid surfaces such that, compared with Surface Evolver simulations, the computing speed is greatly improved. Finally, by comparing the results of the finite element simulations performed with Surface Evolver with those of the method employed in this paper, the practicality of the method is demonstrated.
\end{abstract}

\section{Introduction}

The capillary bridge between two structured surfaces is very important in research into, and application of, micromechanical structures, surface mount technologies, microfluid dynamics, biological bionics, and other technical fields (Stewart et al., 2013; Bowden, 1997; Gau et al., 1999; Mlota et al., 2010; Saad and Neumann, 2014). The forces exerted by the liquid bridge have a great effect on the life and reliability of microelectromechanical systems (MEMS), flip-chip alignment, and the mobility of fluids in micro-channels (Guo et al., 2009; Chen and Soh, 2008; Bush et al., 2010; Dalin et al., 2010; Krammer, 2014). Therefore, research into the mechanical properties of a liquid bridge has great practical significance. By using micro-contact printing, vapor deposition, photolithography, and other techniques, a hydrophobic substrate surface can be patterned with hydrophilic strips to produce a structured surface, so as to control the morphology of the capillary bridge and achieve the desired mechanical properties. This has a high application value in fields such as microelectronics, semiconductors, MEMS, and microchannels (Bowden, 1997; Gau et al., 1999; Bush et al., 2010;
Lipowsky, 2001; Broesch and Frechette, 2012; Broesch et al., 2013).

The capillary bridge between two structured surfaces can be used as a simplified model for biological adhesives, microfluidic channels, and self-assembly (Gau et al., 1999; Bush et al., 2010; De Souza et al., 2008; Broesch et al., 2014; Mermoz et al., 2012; Ferraro et al., 2012; Luo et al., 2014). In particular, a structured surface has the shape characteristics of a slender rectangle, which has great potential for application to the field of microchannels (Gau et al., 1999; Valencia et al., 2001; Lipowsky et al., 2000). Some studies have expressed concern about the effect of these slender rectangular structured surfaces. Some of these studies (Broesch and Frechette, 2012; Broesch et al., 2013) have addressed the relationship between the force of the capillary bridge between two slender structured surfaces and the morphological evolution of a liquid-gas interface, but the conclusions drawn could not be applied to situations where the length of the capillary is confined. One study (Swain and Lipowsky, 2000) addressed a slab geometry with a wetting phase confined between two chemically patterned substrates, but the results obtained through 2-D analysis did not correspond to 


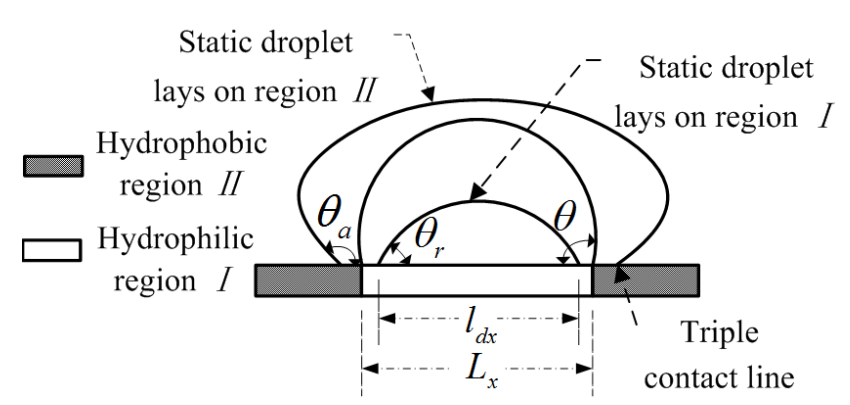

Figure 1. Change in contact angle $\theta$ caused by differences in wettability.

the actual situation. Another study (Valencia et al., 2001) addressed the morphology of the liquid phases within chemically structured slit pores, using the finite element method to evolve the surface towards minimal energy, but this suffered from the trade-off between computing speed and accuracy. Surface Evolver uses the gradient descent method to minimize the free energy of the system, and can be applied to a wide range of problems, rather than being optimized to deal with a specific problem (Brakke, 1994). To obtain a precision solution, therefore, it is necessary to progressively refine the surfaces by applying a tessellated mesh and allowing more time for the computing. Surface Evolver has some shortcomings, such as the fact that surfaces are allowed to intersect with each other and convergence to a minimum energy level can be difficult to determine (Brakke, 1994). Therefore, there is a need for a high-efficiency method capable of overcoming these issues.

We adopted the method of equivalent tension to establish a pseudo-three-dimensional force model for the capillary bridge. Considering the marginal effects of the slender capillary bridge profile and the Young-Laplace equation, we set out to solve the differential equations defining the shape of a capillary bridge having a constant volume. To maximize the efficiency with which the initial-value problem can be solved, an efficient mathematical analysis method is used to solve the boundary-value differential equations for the capillary bridge profiles, therefore optimizing the initial value. Finally, the relationship between the forces and the shape parameters of the capillary bridge is explored. For every calculation, we employ the equation defining the equilibrium of the forces to solve the differential equations, then the change processes for the capillary bridge shape parameters are obtained by means of a decline process defining the separation between the plates, which satisfies the physical boundary constraints. As there is no need to consider meshing liquid surfaces, the computing speed is greatly improved relative to the simulations performed with Surface Evolver. Moreover, there are no shortcomings, such as surfaces intersecting with each other or convergence to a minimum energy being difficult to judge.

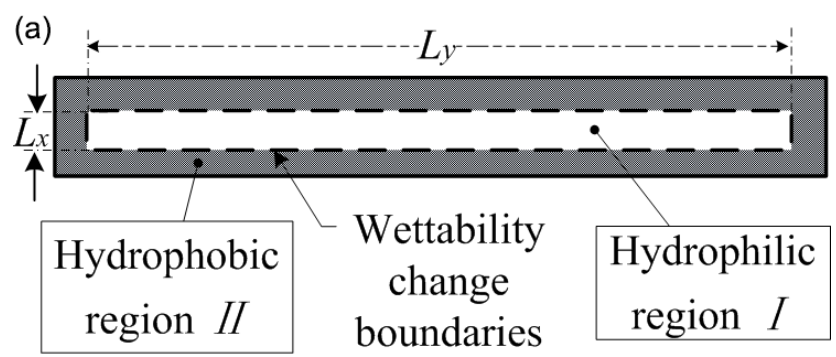

(b)

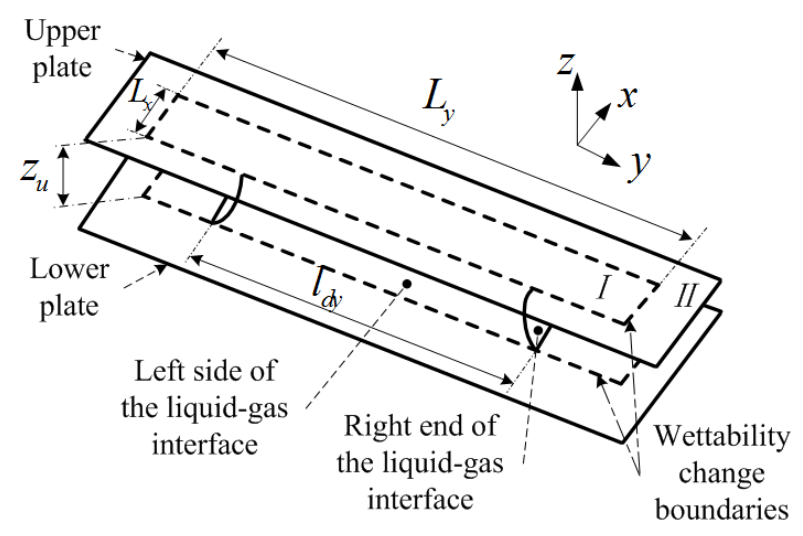

Figure 2. (a) Structured surface of the plate and (b) capillary bridge.

\section{Characteristic parameters and force analysis for the capillary bridge}

\subsection{Characterizations}

On a smooth homogeneous surface, the contact angle between a liquid-gas interface and a liquid-solid interface remains constant. This is known as the "wetting angle" (Langbein, 2002; Bonn et al., 2009). Under a specific gas atmosphere, the size of the wetting angle is affected only by the surface tension between the liquid and the solid (Langbein, 2002; Bonn et al., 2009; Pozrikidis, 2012; Oliver et al., 1977). As shown in Fig. 1, when the triple contact line slips on the structured surface and reaches the point where the wettability changes, then the triple contact line will be pinned and the contact angle will be changed. This is known as "canthotaxis" (Lipowsky et al., 2000; Herminghaus et al., 2008; Liu et al., 2012; Langbein, 2002). If the liquid is static in region I or II, then the contact angles will be $\theta_{r}$ or $\theta_{a}$, respectively. The variation in the contact angle $\theta$ is: $\theta_{r} \leq \theta \leq \theta_{a}$ when $l_{d x}=L_{x}, \theta=\theta_{r}$ when $l_{d x}<L_{x}$, and $\theta=\theta_{a}$ when $l_{d x}>L_{x}$.

As shown in Fig. 2, the slender capillary bridge consists of two plates parallel to each other, both of which have slender rectangular structured surfaces. The structured surface is produced by depositing hydrophilic strips (region I) on a substrate with a hydrophobic surface (region II) through different techniques (Gau et al., 1999; Lipowsky, 2001; Broesch 
and Frechette, 2012; Broesch et al., 2013; Valencia et al., 2001) (see Fig. 2a). The length of region I is set to $L_{y}$ and its width to $L_{x}$. Region I has a high aspect ratio $\left(L_{y} \gg L_{x}\right)$. In this paper we only consider a capillary bridge confined within the region I, that is, the case where $l_{d x}=L_{x}$. Ignoring the roughness of the structured surfaces and evaporation from the capillary bridge, a change in the spacing between the plates causes the two ends of the capillary bridge to advance or recede along the length of the region I, while the contact angles at both ends of the capillary bridge remain constant (Broesch and Frechette, 2012; Broesch et al., 2013; Valencia et al., 2001; Swain and Lipowsky, 2000). This is termed the "slipping" of the triple contact lines on region I. The widths $\left(l_{d x}\right)$ of the liquid-solid interfaces are confined by the hydrophobic region II and are constant, but the contact angle and the curvature of the lateral liquid-gas interface along the length of the hydrophilic region I will vary with the decline in the capillary bridge height, termed the "hinge movement" of the bilateral liquid-gas interfaces at the location where the wettability varies (Broesch and Frechette, 2012; Broesch et al., 2013; Valencia et al., 2001; Yaneva et al., 2005). As the spacing between the plates decreases further, the triple contact line at the two ends of the capillary bridge slips and touches region II, at which point the triple contact line is pinned and the all-around liquid-gas interface of the capillary bridge hinges, which inevitably leads to variations such as the forces of the capillary bridge, internal pressure, surface curvature, and contact angles (Lipowsky, 2001; Broesch and Frechette, 2012; Broesch et al., 2013).

\subsection{Force analysis for capillary bridge}

Based on the three-dimensional shape characteristics of the capillary bridge, a pseudo-three-dimensional force model of the capillary bridge was developed by applying the tension equivalent method. In the Cartesian coordinate system, the upward direction of the force is positive. As shown in Fig. 2, taking advantage of the symmetry of the capillary bridge, the force analysis of the right end and the right side of the liquidgas interfaces is represented by Fig. $3 a$ and $b$.

The hydrostatic equilibrium equation of the capillary bridge in the vertical direction can be written as

$W_{z}+l_{d x} l_{d y} p_{b}-\rho g V_{0}-2 T\left(l_{d x} \sin \theta_{d i}+l_{d y} \sin \theta_{d j}\right)=0$.

At the bottom of the capillary bridge, the pressure is

$p_{b}=\frac{2 T\left(l_{d x} \sin \theta_{d i}+l_{d y} \sin \theta_{d j}\right)+\rho g V_{0}-W_{z}}{l_{d x} l_{d y}}$

where $\rho$ is the density of the liquid and $T$ is the surface tension of the liquid-gas interface. The length of the liquidsolid interface on the lower plate is set to $l_{d y}$, while its width is set to $l_{d x} . \theta_{d i}$ and $\theta_{d j}$ are the contact angles between the liquid-gas interface and the liquid-solid interface along the width and length of the liquid bridge, respectively. $V_{0}$ represents the volume of the liquid; $W_{z}$ represents the total force

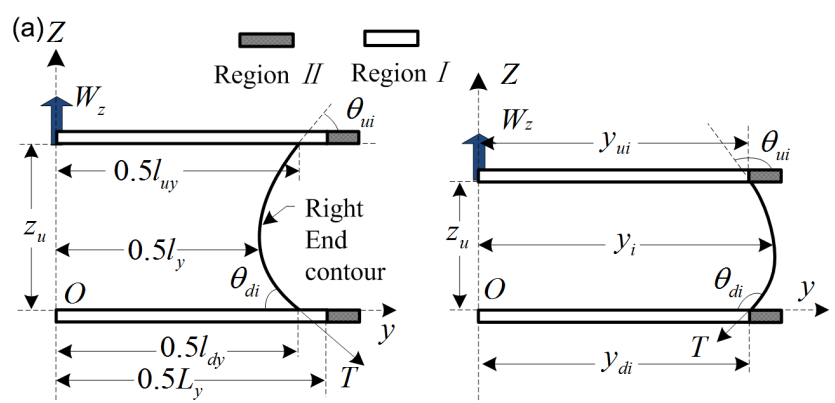

(b)
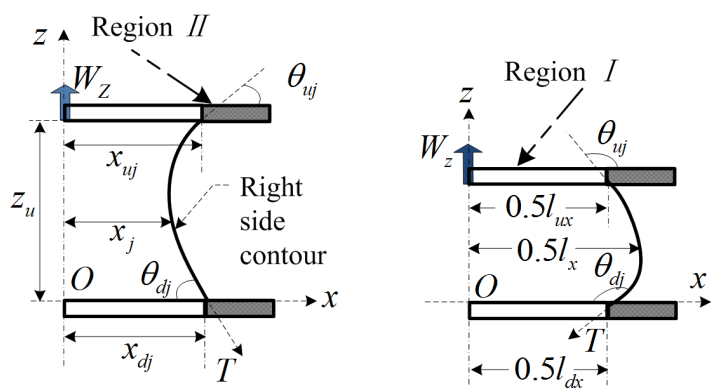

Figure 3. (a) Right end of liquid-gas interface force diagram with different spacings and (b) lateral liquid-gas interface force diagram with different spacings.

applied to the top of the capillary bridge by the upper plate in the vertical direction, which involves the capillary force and the vertical component of the surface tension.

\section{Differential equations defining capillary bridge characterizations}

\subsection{Transformation of Young-Laplace equation}

Unknowns $W_{z}$ and $p_{b}$ cannot be solved using Eqs. (1) and (2), but the hydrostatic equilibrium equation shows that the unknowns are closely related to the shape characteristic parameters of the capillary bridge. To solve unknowns $W_{z}$ and $p_{b}$, solving the differential equations defining the capillary bridge shape becomes key to the problem. Assuming that the liquid volume is constant and transforms the YoungLaplace equation (Padday, 1971), we can develop differential equations to define the shape of the capillary bridge. Because of the high aspect ratio of the capillary bridge $\left(l_{y} \gg l_{x}\right.$, where $l_{y}$ and $l_{x}$ are the length and width of the capillary bridge, respectively, as shown in Fig. 3), the bilateral curved surfaces of the liquid-gas interface can be regarded as approximating to cylindrical surfaces. Then, one of the major radiuses of curvature of the Young-Laplace equation

$1 / \gamma+1 / \gamma^{\prime}=\Delta p / T$

approximates to infinity $\left(\gamma^{\prime} \Rightarrow \infty\right)$. The curvature of the bilateral curved surfaces can thus be written as:

$1 / \gamma=\Delta p / T$ 
where $\Delta p$ is the Laplace pressure. We can employ the surrounding atmospheric pressure as a reference $\left(\Delta p=p_{b}-p_{z}\right)$, where $p_{z}=\rho g z$ represents the variation in the internal pressure due to gravity, and $p_{b}$ is the pressure at the bottom of the capillary bridge.

The top view at the right end of the slender capillary bridge is shown in Fig. 4. Given the slender shape of the capillary bridge, the major radius of curvature of the two-end curved surfaces can be approximated as being equal to half the capillary bridge width $l_{x}$. Based on Eq. (3), the curvature of the two-end curved surfaces can be expressed as:

$1 / \gamma=\Delta p / T-1 / \gamma^{\prime}$

where $\gamma^{\prime}=x_{j}, x_{j}=0.5 l_{x}$ and the capillary bridge width $l_{x}$ varies with the height coordinate $z$.

As shown in Fig. 3, the height coordinate $z$ is chosen as an independent variable in order to satisfy the coordinating relationship between the shape characteristic parameters and pressure, such that the differential expressions of the characteristic parameters can be obtained. For different separations between the plates, the volume differential equation for $z$ is obtained by assuming that the capillary bridge volume is constant. The differential equations for the shape characteristic parameters can thus be written as follows:

$$
\left\{\begin{aligned}
\frac{\mathrm{d} \theta_{i}}{\mathrm{~d} z} & =\frac{1}{\sin \theta_{i}}\left(\frac{\Delta p}{T}-\frac{1}{\gamma^{\prime}}\right) \\
\frac{\mathrm{d} y_{i}}{\mathrm{~d} z} & =1 / \tan \theta_{i} \\
\frac{\mathrm{d} \theta_{j}}{\mathrm{~d} z} & =\frac{\Delta p}{T \sin \theta_{j}} \\
\frac{\mathrm{d} x_{j}}{\mathrm{~d} z} & =1 / \tan \theta_{j} \\
\frac{\mathrm{d} V}{\mathrm{~d} z} & =4 x_{j} y_{i}-2\left(2 x_{j}^{2}-\frac{\pi x_{j}^{2}}{2}\right)
\end{aligned}\right.
$$

where suffixes $i$ and $j$ are the labels of both ends and the bilateral liquid-gas interface profile curves, respectively; $z \in\left[0, z_{u}\right]$, where $z_{u}$ is the separation between the plates (the height of the capillary bridge in the stable state), $\theta_{i}$ and $\theta_{j}$ are the slope angles of the outline curves in the yoz and $x o z$ planes, respectively, and $y_{i}$ and $x_{i}$ are the abscissas of the outline curves in the yoz and $x o z$ planes, respectively (see Fig. 3).

\subsection{Boundary conditions of capillary bridge between slender plates}

Based on the constraints and the characterization of the capillary bridge, the boundary values for the bottom and top of both ends and the bilateral liquid-gas interface profile curves are related as follows:

1. when $z=z_{u}$,

$$
\begin{aligned}
& x_{j}\left(z_{u}\right)=0.5 l_{u x}=0.5 L_{x} ; \theta_{j}\left(z_{u}\right)=\theta_{u j} ; \\
& \theta_{i}\left(z_{u}\right)=\theta_{u i} ; y_{i}\left(z_{u}\right)=0.5 l_{u y} ; V\left(z_{u}\right)=V_{0},
\end{aligned}
$$

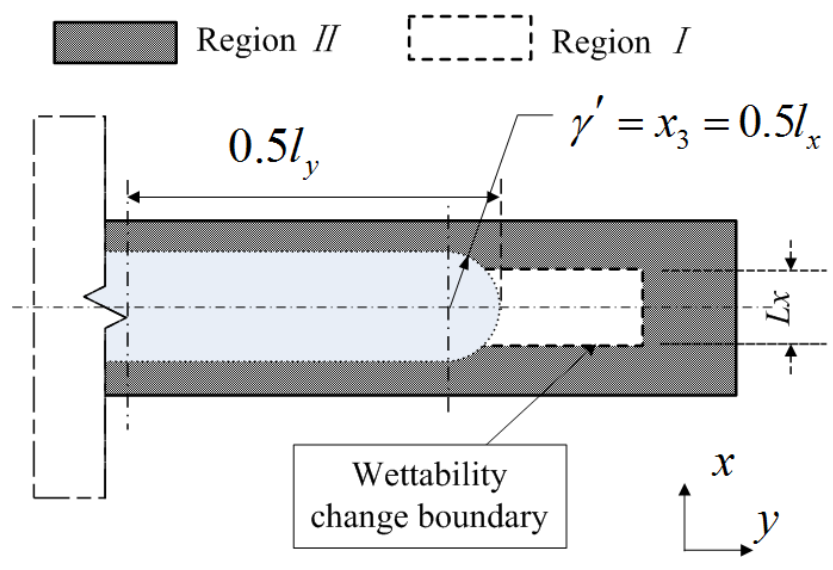

Figure 4. Top view of right end of capillary bridge.

2. when $z=0$,

$$
\begin{aligned}
& x_{j}(0)=0.5 l_{d x}=0.5 L_{x} ; \theta_{j}(0)=\theta_{d j} ; \theta_{i}(0)=\theta_{d i} ; \\
& y_{i}(0)=0.5 l_{d y} ; V(0)=0,
\end{aligned}
$$

where $\theta_{d j} \in\left[\theta_{r}, \theta_{a}\right]$. The length of the liquid-solid interface on the capillary bridge top is set to $l_{u y}$, and its width to $l_{u x}$. When the triple contact lines at the two ends of the capillary bridge have not yet touched region II, $\theta_{i}\left(z_{u}\right)=\theta_{u i}=\theta_{r}, \theta_{i}(0)=\theta_{d i}=\theta_{r}$. When the triple contact lines touch the ends of the region $\mathrm{I}, y_{i}\left(z_{u}\right)=0.5 l_{u y}=0.5 L_{y}$, $y_{i}(0)=0.5 l_{d y}=0.5 L_{y}$.

The solution to differential Eq. (6) is equivalent to solving for the boundary values of first-order nonlinear differential equations, obtaining five unknowns with only one independent variable $z\left(z \in\left[0, z_{u}\right]\right)$. Because of the mutual coupling between the contact angle $\theta_{d i}$ and $\theta_{d j}$ at the bottom of the capillary bridge, differential Eq. (6) can thus be solved.

\section{Methods for solving differential equations}

\subsection{Making the boundary-value problem equal to an initial-value problem}

To maximize the efficiency with which an initial-value problem can be solved, the boundary-value conditions for Eq. (8) are assumed to be equivalent to the initial-value conditions, but the solution to the initial-value problem cannot satisfy the boundary-value of Eq. (7), which becomes a problem of objective function minimization. Obtaining the boundary-value conditions for Eq. (7) is equivalent to minimizing relative error objective functions (Eqs. 9 and 10).

When $l_{d y}<L_{y}, \theta_{i}\left(z_{u}\right)=\theta_{i}(0)=\theta_{r}$. The objective function can then be written as 


$$
\begin{aligned}
\operatorname{Min}: & F\left(\theta_{d j}^{*}, l_{d y}^{*}, W_{z}^{*}\right)=\frac{\left(\theta_{i}\left(z_{u}\right)-\theta_{r}\right)^{2}}{\left(\theta_{r}\right)^{2}} \\
& +\frac{\left(x_{j}\left(z_{u}\right)-0.5 L_{x}\right)^{2}}{\left(0.5 l_{d x}\right)^{2}}+\frac{\left(V\left(z_{u}\right)-V_{0}\right)^{2}}{V_{0}^{2}}
\end{aligned}
$$

which includes the implied variables such as bilateral contact angles $\theta_{d j}^{*}$, the length $l_{d y}^{*}$ at the bottom of the liquid-solid interface and the force $W_{z}^{*}$ on the top of the capillary bridge to be optimized. $\left[z_{u 2}^{*}, z_{u 1}^{*}\right]$ are the spacing ranges between the plates (capillary bridge height $z_{u}$ ) satisfying boundaryvalue conditions for Eqs. (7) and (8). If the spacing between the plates is greater than $z_{u 1}^{*}$, there is a tendency for the bilateral contact angles $\theta_{d j}^{*}>\theta_{a}$. Thus, the triple contact lines constituted by the bilateral liquid-gas interfaces and plates will be free from restraint and slide into region II. When the triple contact lines at the end of the capillary bridge touch region II, the separation between the plates is $z_{u 2}^{*}, l_{d y}=L_{y}$ and $\theta_{i}\left(z_{u}\right)=\theta_{i}(0)=\theta_{r}$. With a further reduction in the height $z_{u}$, the liquid-gas surfaces of both sides and two ends will hinge at the same time.

When $l_{d y}=L_{y}, \quad y_{i}(0)=0.5 l_{d y}=0.5 L_{y}$. The objective function is as follows:

$$
\begin{aligned}
\operatorname{Min}: & F\left(\theta_{d i}^{*}, \theta_{d j}^{*}, W_{z}^{*}\right)=\frac{\left(y_{i}\left(z_{u}\right)-0.5 L_{y}\right)^{2}}{\left(0.5 l_{d y}\right)^{2}} \\
& +\frac{\left(x_{j}\left(z_{u}\right)-0.5 L_{x}\right)^{2}}{\left(0.5 l_{d x}\right)^{2}}+\frac{\left(V\left(z_{u}\right)-V_{0}\right)^{2}}{V_{0}^{2}}
\end{aligned}
$$

which includes the implied variables, such as the contact angles of the two ends $\theta_{d i}^{*}$, the bilateral contact angles $\theta_{d j}^{*}$ and the force $W_{z}^{*}$ on the top of the capillary bridge, which has to be optimized. Here, $\left[z_{u 3}^{*}, z_{u 2}^{*}\right]$ are the capillary bridge heights $z_{u}$ satisfying boundary-value conditions for Eqs. (7) and (8). If the spacing between the plates is less than $z_{u 3}^{*}$, the triple contact lines will be free from restraint and slide into region II.

For a height range $z_{u} \in\left[z_{u 3}^{*}, z_{u 1}^{*}\right]$, as the height $z_{u}$ is reduced, there are $m$ capillary bridge heights $\left(z_{u}=z_{u}(0)\right.$, $\left.z_{u}(1), \cdots, z_{u}(m)\right)$. Under those constraint conditions in which the capillary bridge could degrade, there are $m$ values of bilateral contact angles. When $z=z_{u}$, the implicit variables are obtained by optimizing the implicit variables (if $l_{d y}<L_{y}$, the implicit variables are $\theta_{d j}^{*}, l_{d y}^{*}$ and $W_{z}^{*}$; if $l_{d y}=L_{y}$, the implicit variables are $\theta_{d i}^{*}, \theta_{d j}^{*}$ and $W_{z}^{*}$ ) in order to attain the permissible errors of the objective function (Eqs. 9 or 10), and to make the boundary-values at the top end of the capillary bridge satisfy Eq. (7).

The flowchart for solving differential Eq. (6) is shown in Fig. 5.

\subsection{Range of capillary bridge height}

If the boundary values at the top and bottom of the outline curves satisfy Eqs. (7) and (8), the method intro-

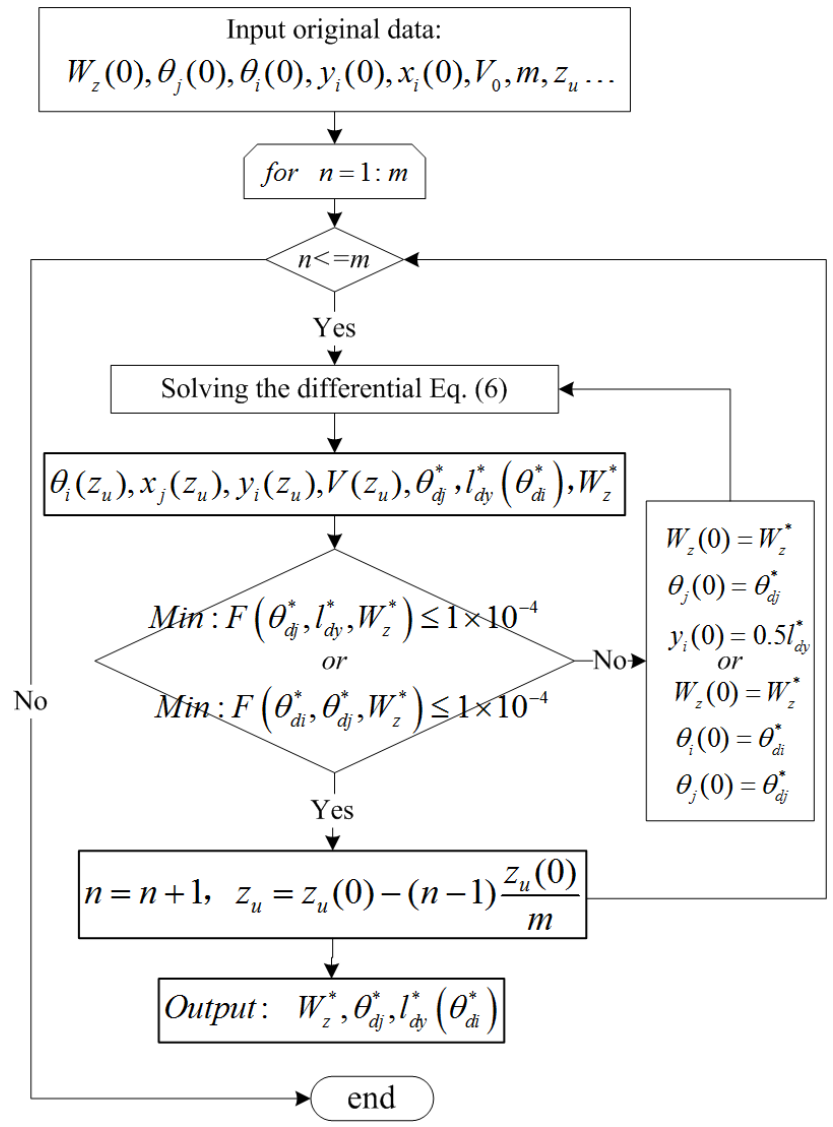

Figure 5. Flowchart for solving differential equations.

duced in Sect. 4.1 can be employed to calculate the spacing ranges between the plates, provided the liquid does not overflow into hydrophobic region II. The height $z_{u 1}^{*}$ which satisfies the constraint conditions can be obtained by optimizing objective function (Eq. 11), when $\theta_{j}(0)=\theta_{d j}=\theta_{a}$ and $l_{d y}<L_{y}$. The height $z_{u 2}^{*}$ can be obtained by optimizing objective function (Eq. 12), when $\theta_{i}\left(z_{u}\right)=\theta_{i}(0)=\theta_{r}$ and $y_{i}\left(z_{u}\right)=0.5 l_{u y}=0.5 L_{y}$. The height $z_{u 3}^{*}$ which satisfies the constraint conditions can be obtained by optimizing objective function (Eq. 13), when $\theta_{j}(0)=\theta_{d j}=\theta_{a}$ and $y_{i}\left(z_{u}\right)=0.5 l_{u y}=0.5 L_{y}$.

$$
\begin{aligned}
\operatorname{Min}: & F\left(z_{u 1}^{*}, l_{d y}^{*}, W_{z}^{*}\right)=\frac{\left(\theta_{i}\left(z_{u 1}^{*}\right)-\theta_{r}\right)^{2}}{\theta_{r}^{2}} \\
& +\frac{\left(x_{j}\left(z_{u 1}^{*}\right)-0.5 l_{d x}\right)^{2}}{\left(0.5 l_{d x}\right)^{2}}+\frac{\left(V\left(z_{u 1}^{*}\right)-V_{0}\right)^{2}}{V_{0}^{2}}
\end{aligned}
$$

$$
\begin{aligned}
\operatorname{Min}: & F\left(z_{u 2}^{*}, \theta_{d j}^{*}, W_{z}^{*}\right)=\frac{\left(\theta_{i}\left(z_{u 2}^{*}\right)-\theta_{r}\right)^{2}}{\theta_{r}^{2}} \\
& +\frac{\left(x_{j}\left(z_{u 2}^{*}\right)-0.5 l_{d x}\right)^{2}}{\left(0.5 l_{d x}\right)^{2}}+\frac{\left(V\left(z_{u 2}^{*}\right)-V_{0}\right)^{2}}{V_{0}^{2}}
\end{aligned}
$$


Table 1. Parameters for capillary bridge and flat plate.

\begin{tabular}{llllcccc}
\hline Parameters & $\begin{array}{l}L_{x} \\
(\mathrm{~mm})\end{array}$ & $\begin{array}{l}V_{0} \\
(\mu \mathrm{L})\end{array}$ & $\begin{array}{c}L_{y} \\
(\mathrm{~mm})\end{array}$ & $\begin{array}{c}\text { Wetting } \\
\text { angles } \theta_{r}\end{array}$ & $\begin{array}{c}\text { Wetting } \\
\text { angle } \theta_{a}\end{array}$ & $\begin{array}{c}\text { Density } \rho \\
\left(\mathrm{kg} \mathrm{mm}^{-3}\right)\end{array}$ & $\begin{array}{c}\text { Surface } \\
\text { tension } T \\
\left(\mathrm{~N} \mathrm{~mm}^{-1}\right)\end{array}$ \\
\hline Group $a$ & 1 & 5 & 10 & $30,45,60^{\circ}$ & $135^{\circ}$ & $1 \times 10^{-6}$ & $7.2 \times 10^{-5}$ \\
Group $b$ & 1 & 7.5 & 10 & $30,45,60^{\circ}$ & $135^{\circ}$ & $1 \times 10^{-6}$ & $7.2 \times 10^{-5}$ \\
Group $c$ & 0.5 & 5 & 10 & $30,45,60^{\circ}$ & $135^{\circ}$ & $1 \times 10^{-6}$ & $7.2 \times 10^{-5}$ \\
\hline
\end{tabular}

$$
\begin{aligned}
\operatorname{Min}: & F\left(z_{u 3}^{*}, \theta_{d i}^{*}, W_{z}^{*}\right)=\frac{\left(y_{i}\left(z_{u 3}^{*}\right)-0.5 L_{y}\right)^{2}}{\left(0.5 L_{y}\right)^{2}} \\
+ & \frac{\left(x_{j}\left(z_{u 3}^{*}\right)-0.5 l_{d x}\right)^{2}}{\left(0.5 l_{d x}\right)^{2}}+\frac{\left(V\left(z_{u 3}^{*}\right)-V_{0}\right)^{2}}{V_{0}^{2}}
\end{aligned}
$$

Objective functions (Eqs. 11 to 13) are implied optimization variables: $z_{u 1}^{*}, l_{d y}^{*}, W_{z}^{*} ; z_{u 2}^{*}, \theta_{d j}^{*}, W_{z}^{*}$ and $z_{u 3}^{*}, \theta_{d i}^{*}, W_{z}^{*}$.

\section{Results and discussion}

Three groups of liquid bridge systems are listed in Table 1. The hydrophilic regions I for different wetting angles, the curves of the morphological parameters, the forces, and internal pressures for a range of heights are analyzed. Moreover, the results are compared with the Surface Evolver simulations.

\subsection{Relationships between morphological parameters}

The outline curves satisfying boundary-value conditions for Eqs. (7) and (8) are obtained by programming for solving differential Eq. (6). Figures 6 and 7 show the outline curves for $V_{0}=5 \mu \mathrm{L}, L_{x}=1 \mathrm{~mm}$, and $\theta_{r}=30^{\circ}$.

When the range of the spacing between the two plates is $z_{u} \in\left[z_{u 2}^{*}, z_{u 1}^{*}\right]$, the triple contact lines of the two ends do not touch hydrophobic region II $\left(l_{d y}<L_{y}\right)$. In Fig. 6a, both sides of the liquid-gas interface gradually change from convex to concave and the contact angles $\theta_{d j}$ decrease with the height $z_{u}$. However, the width at the bottom of the capillary bridge is constant $\left(l_{d x}=L_{x}\right)$, because the bilateral triple contact lines are pinned by hydrophobic region II along the length of region I, and the bilateral liquid-gas interfaces are in a "hinge movement" state. In Fig. $6 \mathrm{~b}$, the contact angles $\theta_{d i}$ of the capillary bridge's two ends are constant $\left(\theta_{d i}=\theta_{r}\right)$, but the spacing between the two plates can vary. The length of the capillary bridge can change freely, the contour surfaces of its two ends are always concave, and the triple contact lines of the two ends "slip" in region I. As shown in Fig. 6b, it can be found that gravity has a significant effect on the morphology of the capillary bridge (there is a large difference between abscissas $y(0)$ and $y\left(z_{u}\right)$ of the upper and lower endpoints $a_{n}$ and $b_{n}$ of the contour curves), when the height of the capillary bridge is greater $\left(z_{u}>1.25 \mathrm{~mm}\right)$. When $z_{u}<1.25 \mathrm{~mm}$,
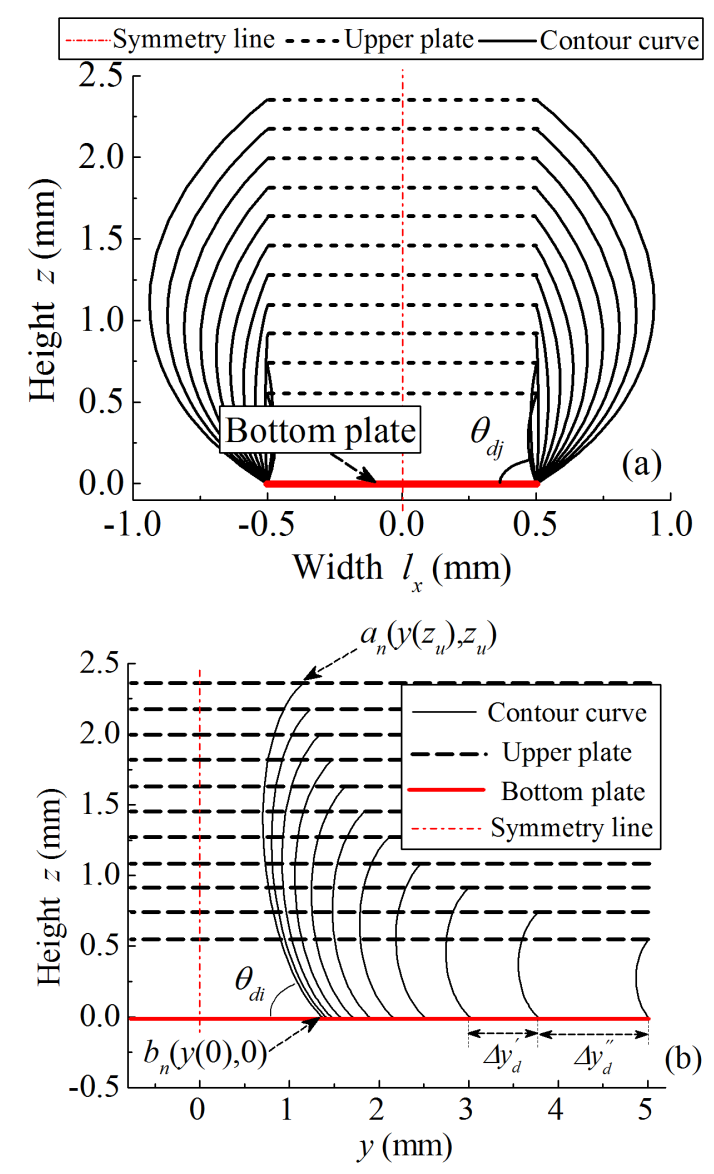

Figure 6. Profiles of the capillary bridge when the two ends' triple contact lines are unconstrained. (a) Bilateral side contour curves and (b) right end contour curves.

$\left|y(0)-y\left(z_{u}\right)\right| \Rightarrow 0$, gravity has an increasingly smaller influence on the morphology of the capillary bridge. The contour curves of the capillary bridge will approximate to a circular arc.

When the height $z_{u} \in\left[z_{u 3}^{*}, z_{u 2}^{*}\right]$, the triple contact lines are also pinned by hydrophobic region II $\left(l_{d y}=L_{y}\right)$ at the two ends of region I. In Fig. 7, the liquid-gas interfaces around the capillary bridge are in a "hinge movement" state and gradually bulge outward.

For different volumes $V_{0}$ or widths $L_{x}$, the bottom lengths of the liquid-solid interfaces are as shown in Fig. 8, when 

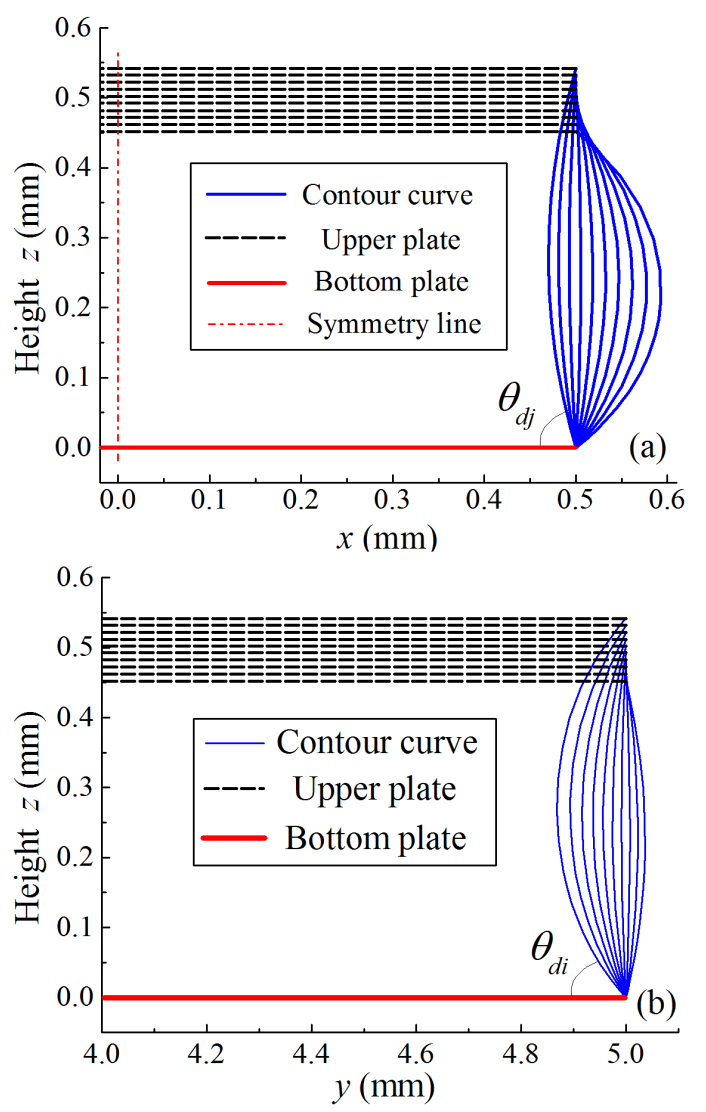

Figure 7. Profiles of capillary bridge when triple contact lines are pinned. (a) Right-side contour curves and (b) right-end contour curves.

the triple contact lines of the two ends "slip" in region I as the height $z_{u}$ falls. It can be found that the lengths of the liquid-solid interfaces are in quantitative agreement with the result of the Surface Evolver simulations when the height $z_{u}$ is low, but as the height increases, we find that the results from our theory underestimate the simulation results. When the height of the capillary bridge is greater $\left(z_{u} / L_{x}>1.5\right)$, we assume that the deviation observed at higher values of $z_{u}$ is caused by a decrease in the aspect ratio $\left(l_{y} / l_{x}\right)$ of the bridge, at which point one of the major curvature radiuses of Eq. (3) approximates to infinity $\left(\gamma^{\prime} \Rightarrow \infty\right)$. By integrating Fig. $8 \mathrm{a}$ and $\mathrm{c}$ it can be found that, for the same volume $V_{0}$, a smaller value of $L_{x}$ will greatly reduce the variation in the height $z_{u}\left(z_{u 1}^{*}\right.$ becomes smaller, $z_{u 3}^{*}$ becomes larger).

For variable $V_{0}$ or region I width $L_{x}$, Fig. 9 indicates the variations in the bilateral contact angles $\theta_{d j}$ with the reduction in the height $z_{u}$. Based on the changes in the wettability of region $\mathrm{I}$, the ranges of the contact angles are $\left[\theta_{r}\right.$, $\left.\theta_{a}\right],\left(\theta_{r}\right.$ is 30,45 or $60^{\circ} ; \theta_{a}$ is $\left.135^{\circ}\right)$. When the capillary bridge can freely advance along the length of region I, $\theta_{d j}$ decreases with the height, which is in agreement with the results of experiments described in the literature (Broesch and
Frechette, 2012) as well as those of the Surface Evolver simulations. Once the triple contact lines at the left and right ends of the capillary bridge have been pinned by region II $\left(l_{d y}=L_{y}\right), \theta_{d j}$ will increase as the height $z_{u}$ decreases. As the height of the capillary bridge increases $\left(z_{u} / L_{x}>1.5\right)$, the deviation also increases relative to the results of the Surface Evolver simulations. The variation in the bilateral contact angles $\theta_{d j}$ is in agreement with the results of the Surface Evolver simulations, except when the wetting angle $\theta_{r}=60^{\circ}$ and $L_{x}=0.5 \mathrm{~mm}$ (see the inset in Fig. 9c), because the results of the Surface Evolver simulations showed that the ends of the capillary bridge did not touch region II in the spacing variable range determined using our theory.

By combining Figs. 9 and 10, it can be seen that $\theta_{d j}$ is always greater than $\theta_{d i}\left(\theta_{d j}>\theta_{d i}\right)$, corresponding to the same height $z_{u}$, which implies that a bulge appears on the side of the liquid-gas interfaces first, and then the bilateral triple contact lines depart from pinning and overflow into hydrophobic region II when the height $z_{u}$ is greater than $z_{u 1}^{*}$ or less than $z_{u 3}^{*}$. The contact angle $\theta_{d i}$ remains constant when both ends of the liquid bridge are able to freely "slip". When the two ends of the capillary bridge are constrained by the region II $\left(l_{d y}=L_{y}\right), \theta_{d i}$ will increase. Based on Figs. 9 to 10, the ranges of variable $z_{u}$ are influenced by the changes in the wetting angle $\theta_{r}$, volume $V_{0}$, and the width $L_{x}$ of the region I. For the same group of parameters in Table $1, \theta_{r}$ only influences $z_{u 1}^{*}$, the upper limit on the range of the height $z_{u}$. If $\theta_{r}$ were smaller, $z_{u 1}^{*}$ could attain a greater value. $V_{0}$ mainly influences $z_{u 3}^{*}$, the lower limit on the range of the height $z_{u}$. If $V_{0}$ were smaller, $z_{u 3}^{*}$ could attain a smaller value. The variations in the bilateral contact angles $\theta_{d i}$ are in agreement with the results of the Surface Evolver simulations, except when the wetting angle $\theta_{r}=60^{\circ}$ and $L_{x}=0.5 \mathrm{~mm}$ (see the inset in Fig. 10c), because the Surface Evolver simulations indicate that the ends of the capillary bridge do not touch region II, such that $\theta_{d i}=60^{\circ}$ remains constant.

\subsection{Stiffness characteristics curves of capillary bridge}

The force $W_{z}$ acting on the capillary bridge upper end is related to $z_{u}$. The height of the capillary bridge as determined by the curves known as the "stiffness characteristics curve" of $W_{z}$ is shown in Fig. 11. As $z_{u}$ decreases, the force $W_{z}$ continues to increase, to the point where the triple contact lines at the ends of the capillary bridge move into the "slipping" stage. According to Eq. (1), the change in $W_{z}$ depends on variables $l_{d y}, p_{b}$ and $\theta_{d j}$. As $\sin \theta_{d j}$ and $l_{d y}$ increase, $p_{b}$ decreases (see Fig. 12), thus demonstrating that the variation in $W_{z}$ is mainly a result of the surface tension. As the width of region I decreases, the change in the wettability of region I exerts a greater influence on the change in $W_{z}$ (see Fig. 11c). As $z_{u}$ further decreases, the triple contact lines touch region II, which are pinned. At this point, the all-around liquid-gas interfaces are in the "hinge movement" stage. Here, the pressure $p_{b}$ is rapidly increasing, but 


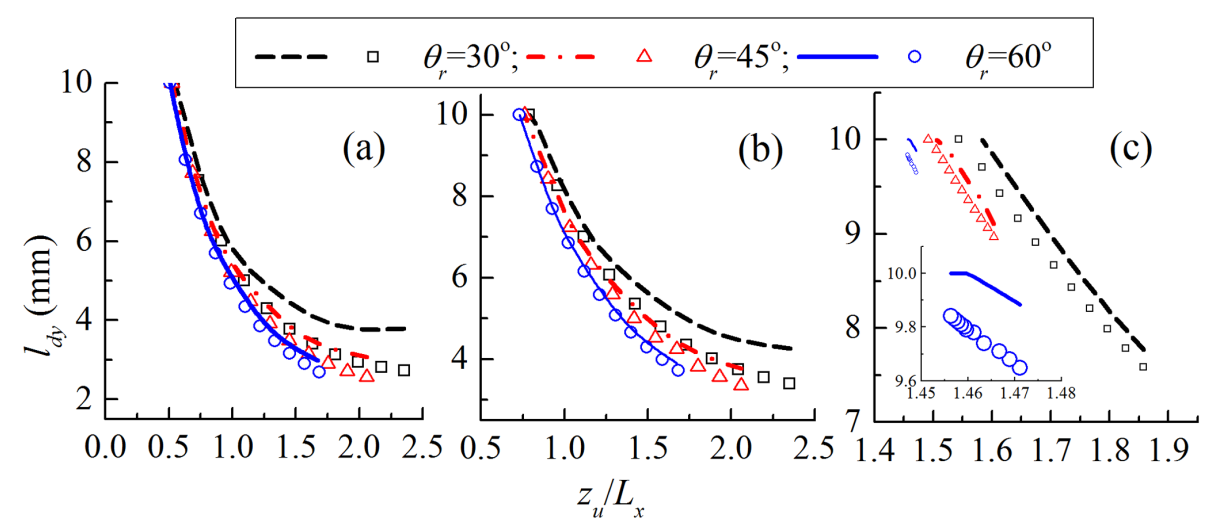

Figure 8. Variations in bottom length of liquid-solid interfaces. (a), (b), and (c) refer to parameters groups $a, b$, and $c$ listed in Table 1 for a range of wetting angles. The data points are as obtained with our theory and the lines are those obtained from the Surface Evolver simulations. The inset shows the change in the bottom length of the liquid-solid interfaces when the wetting angle $\theta_{r}=60^{\circ}$.

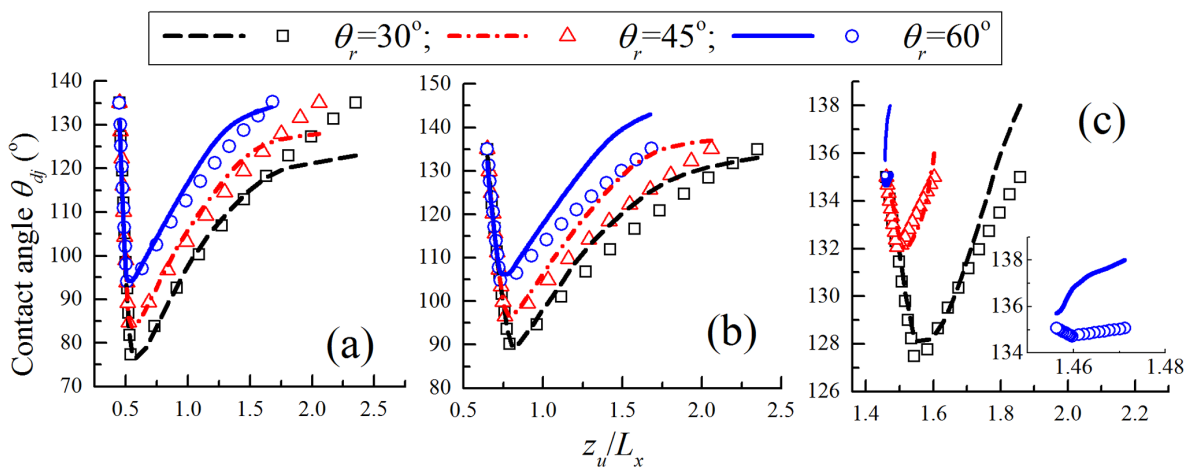

Figure 9. Changes in contact angle $\theta_{d j}$ at both sides. Here, (a), (b), and (c) correspond to parameter groups $a, b$, and $c$ listed in in Table 1 for different wetting angles. The data points are as obtained with our theory and the lines are those obtained from the Surface Evolver simulations.

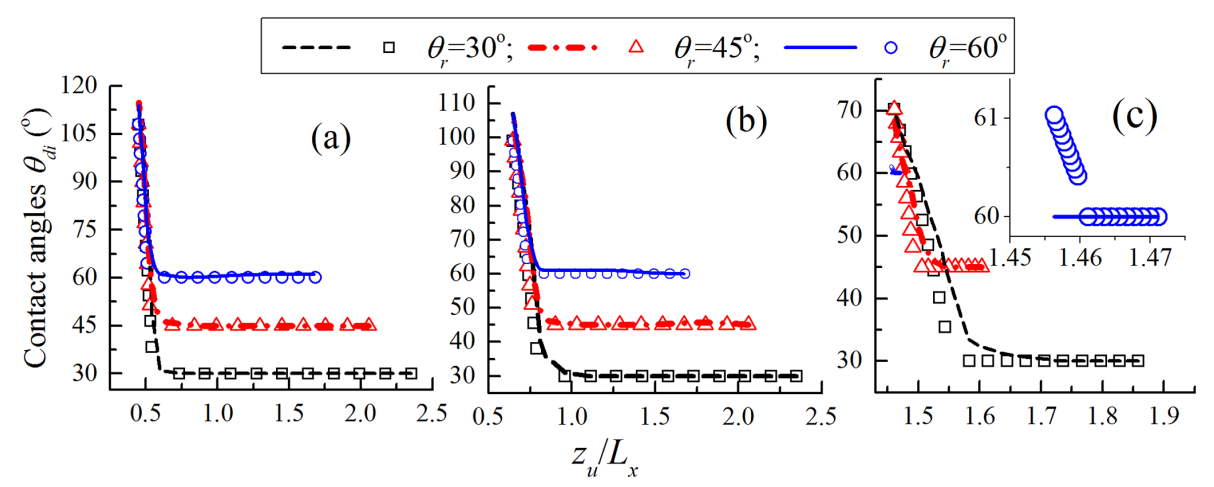

Figure 10. Changes in contact angles $\theta_{d i}$ at both ends. Here, (a), (b), and (c) refer to parameter groups $a, b$, and $c$ that are listed in Table 1 for different wetting angles. The data points are as obtained with our theory and the lines are those obtained from the Surface Evolver simulations.

the force $W_{z}$ exerted on the capillary bridge by the upper plate is rapidly declining and it changes from stress to pressure as the height decreases, which is mainly affected by the capillary force on the capillary bridge. When we compare the predictions obtained from the Surface Evolver simulations with the results obtained with our method, we find that the two sets of results are in quantitative agreement, but as the region I widths $L_{x}=0.5 \mathrm{~mm}$ and wetting angle $\theta_{r}=60^{\circ}$ (see the inset in Fig. 11c), we can see that the results of the Sur- 


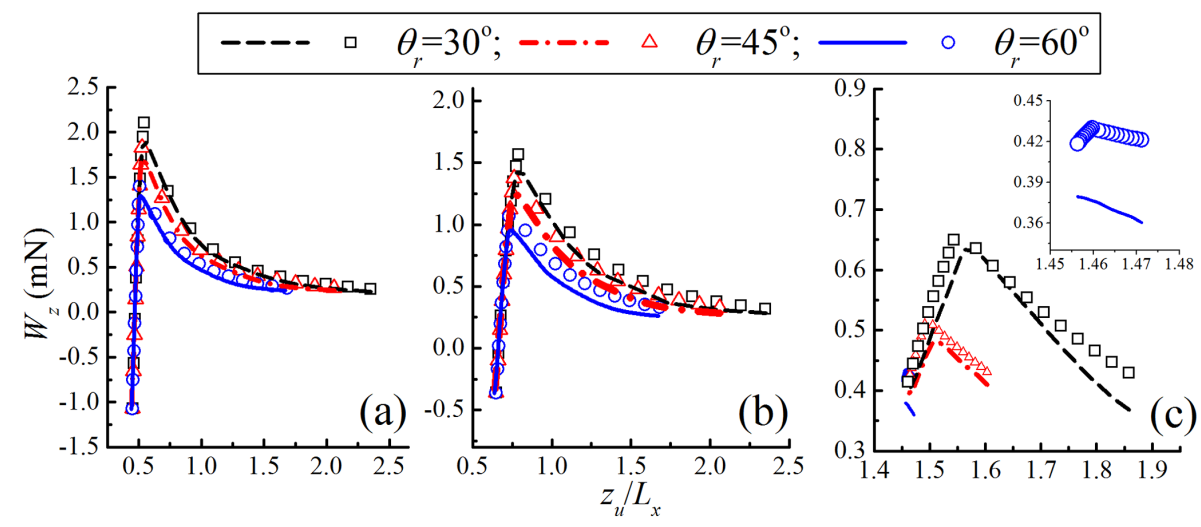

Figure 11. Stiffness characteristic curves of $W_{z}$. Here, (a), (b), and (c) correspond to parameter groups $a, b$, and $c$, listed in Table 1 for different wetting angles. The data points are as obtained with our theory and the lines are those obtained from the Surface Evolver simulations.

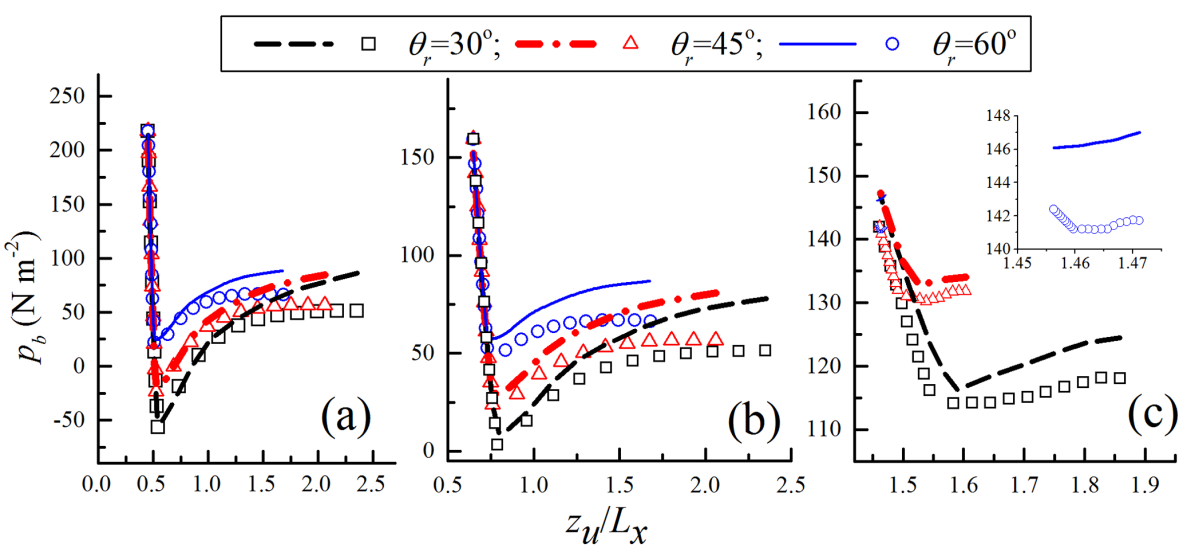

Figure 12. Variations in bottom pressure of capillary bridge. Here, (a), (b), and (c) refer to groups $a, b$, and $c$ listed in Table 1 for different wetting angles. The data points are as obtained with our theory and the lines are those obtained from the Surface Evolver simulations.

face Evolver simulations are about $15 \%$ less than the results obtained with our theory.

Figure 12 shows the changes in the pressure $p_{b}$. When the triple contact lines are "slipping", the decline in the upper plate causes the capillary bridge to elongate. When the value of $p_{b}$ is falling slowly, a transition from negative to positive values at around $z_{u} / L_{x}=1$ can be seen for parameter group $a$ and the wetting angle $\theta_{r}=30^{\circ}$. This corresponds directly to the transition in the contact angles $\theta_{d j}$ from greater than $90^{\circ}$ to less than $90^{\circ}$, as shown in Fig. 9a. When the all-around liquid-gas interfaces are in the "hinge movement" stage, the capillary bridge does not elongate any further. The descent of the upper plate causes the internal pressure to increase rapidly. This corresponds to a variation in the contact angles $\theta_{d j}$ and $\theta_{d j}$, shown in Figs. 9 and 10. When the height of the capillary bridge is greater $\left(z_{u} / L_{x}>1.5\right)$, we can see that the results of our theory underestimate the results obtained with the Surface Evolver simulations, due to the breakdown of the Eqs. (4) and (5) approximation, as discussed in the previous sections.

\section{Conclusions}

A pseudo-three-dimensional force model of a capillary bridge was developed by the application of the tension equivalent method based on the three-dimensional shape characteristic of the capillary bridge. First, the rules governing the capillary bridge's characteristic parameters were identified by integrating the initial values and final values of differential equations for the capillary bridge characteristic parameters and by optimizing the initial values for obtaining the boundary values of differential equations. Then the rules governing the changes of the contact angles were analyzed for the "slipping" and "hinge movement" stages. Further, the stiffness characteristic curves of the capillary bridge were obtained by analyzing the relationship between the forces and the separations between the plates. Finally, the changes in the internal pressure of the capillary bridge were explored. It is found that the rules governing the forces and the capillary bridge's characteristic parameters are in better agreement with the Surface Evolver simulations, and agree especially well for low val- 
ues of $z_{u} / L_{x}$. The theory highlights the fact that the computing speed is greatly improved, relative to the Surface Evolver simulations. Moreover, there are no shortcomings, such as surfaces intersecting with each other or convergence to the minimum energy level being difficult to judge. This is particularly useful for the study of these issues. This would reduce the computing time needed to determine the equilibrium positions when a package with a large number of solder joints is assembled onto a PCB.

Acknowledgements. The authors would like to thank the supports by the National Natural Science Foundation of China (No. 61201021, No. 51306134).

Edited by: A. Eriksson

Reviewed by: two anonymous referees

\section{References}

Bonn, D., Eggers, J., Indekeu, J., Meunier, J., and Rolley, E.: Wetting and spreading, Rev. Mod. Phys., 81, 739-805, 2009.

Bowden, N.: Self-Assembly of Mesoscale Objects into Ordered Two-Dimensional Arrays, Science, 276, 233-235, 1997.

Brakke, K. A.: Surface evolver manual. Mathematics Department, Susquehanna Univerisity, Selinsgrove, PA, 1994.

Broesch, D. J. and Frechette, J.: From Concave to Convex: Capillary Bridges in Slit Pore Geometry, Langmuir, 28, 15548-15554, 2012.

Broesch, D. J., Dutka, F., and Frechette, J.: Curvature of Capillary Bridges as a Competition between Wetting and Confinement, Langmuir, 29, 15558-15564, 2013.

Broesch, D. J., Shiang, E., and Frechette, J.: Role of substrate aspect ratio on the robustness of capillary alignment, Appl. Phys. Lett., 104, 1-5, 2014.

Bush, J. W. M., Peaudecerf, F., Prakash, M., and Quéré, D.: On a tweezer for droplets, Adv. Colloid Interf. Sci., 161, 10-14, 2010.

Chen, S. H. and Soh, A. K.: The capillary force in micro- and nanoindentation with different indenter shapes, Int. J. Solids Struct., 45, 3122-3137, 2008.

Dalin, J., Wilde, J., Zulfiqar, A., Lazarou, P., Synodinos, A., and Aspragathos, N.: Electrostatic attraction and surface-tensiondriven forces for accurate self-assembly of microparts, Microelect. Eng., 87, 159-162, 2010.

De Souza, E. J., Brinkmann, M., Mohrdieck, C., and Arzt, E.: Enhancement of Capillary Forces by Multiple Liquid Bridges, Langmuir, 24, 8813-8820, 2008.

Ferraro, D., Semprebon, C., Tóth, T., Locatelli, E., Pierno, M., Mistura, G., and Brinkmann, M.: Morphological Transitions of Droplets Wetting Rectangular Domains, Langmuir, 28, 1391913923, 2012.

Gau, H., Herminghaus, S., Lenz, P., and Lipowsky, R.: Liquid morphologies on structured surfaces: from microchannels to microchips, Science, 283, 46-49, 1999.
Guo, J.-G., Zhou, L.-J., and Zhao, Y.-P.: Instability analysis of torsional MEMS/NEMS actuators under capillary force, J. Colloid Interf. Sci., 331, 458-462, 2009.

Herminghaus, S., Brinkmann, M., and Seemann, R.: Wetting and dewetting of complex surface geometries, Annu. Rev. Mater. Res., 38, 101-121, 2008.

Krammer, O.: Modelling the self-alignment of passive chip components during reflow soldering, Microelect. Reliabil., 54, 457463, 2014.

Langbein, D.: Canthotaxis/Wetting Barriers/Pinning Lines, in: Capillary Surfaces, Springer Tracts in Modern Physics, Springer, Berlin, Heidelberg, 149-177, 2002.

Lipowsky, R.: Morphological wetting transitions at chemically structured surfaces, Curr. Opin. Colloid Interf. Sci., 6, 40-48, 2001.

Lipowsky, R., Lenz, P., and Swain, P. S.: Wetting and dewetting of structured and imprinted surfaces, Colloids Surf. A, 161, 3-22, 2000.

Liu, J., Xia, R., and Zhou, X.: A new look on wetting models: continuum analysis, Sci. China Phys. Mech. Astron., 55, 2158-2166, 2012.

Luo, C., Heng, X., and Xiang, M.: Behavior of a Liquid Drop between Two Nonparallel Plates, Langmuir, 30, 8373-8380, 2014.

Mermoz, S., Sanchez, L., Di Cioccio, L., Berthier, J., Deloffre, E., and Fretigny, C.: Impact of containment and deposition method on sub-micron chip-to-wafer self-assembly yield, IEEE International 3D Systems Integration Conference (3DIC), Osaka, USA, $1-5,2012$.

Mlota, N. J., Toveyb, C. A., and Hua, D. L.: Fire ants self-assemble into waterproof rafts to survive floods, P. Natl. Acad. Sci., 108, 7669-7673, 2010.

Oliver, J. F., Huh, C., and Mason, S. G.: Resistance to spreading of liquids by sharp edges, J. Colloid Interf. Sci., 59, 568-581, 1977.

Padday, J. F.: The Profiles of Axially Symmetric Menisci, Philos. T. Roy. Soc. Lond. A, 269, 265-293, 1971.

Pozrikidis, C.: Stability of sessile and pendant liquid drops, J. Eng. Math., 72, 1-20, doi:10.1007/s10665-011-9459-3, 2012.

Saad, S. M. I. and Neumann, A. W.: Total Gaussian curvature, drop shapes and the range of applicability of drop shape techniques, Adv. Colloid Interf. Sci., 204, 1-14, doi:10.1016/j.cis.2013.12.001, 2014.

Stewart, M. P., Hodel, A. W., Spielhofer, A., Cattin, C. J., Müller, D. J., and Helenius, J.: Wedged AFM-cantilevers for parallel plate cell mechanics, Methods, 60, 186-194, 2013.

Swain, P. S. and Lipowsky, R.: Wetting between structured surfaces: Liquid bridges and induced forces, Europhys. Lett., 49, 203-209, 2000.

Valencia, A., Brinkmann, M., and Lipowsky, R.: Liquid Bridges in Chemically Structured Slit Pores, Langmuir, 17, 3390-3399, 2001.

Yaneva, J., Milchev, A., and Binder, K.: Polymer droplets on substrates with striped surface domains: molecular dynamics simulations of equilibrium structure and liquid bridge rupture, J. Phys. Condens. Matter, 17, S4199-S4211, doi:10.1088/09538984/17/49/014, 2005. 